\title{
Convergence of Jacobi and Gauss-Seidel Method and Error Reduction Factor
}

\author{
${ }^{1}$ HarpinderKaur, ${ }^{2}$ KhushpreetKaur \\ ${ }^{1,2}$ Department of Mathematics Baba FaridCollege,Bathinda
}

\begin{abstract}
In this paper, it is shown that neither of the iterative methods always converges. That is, it is possible to apply the Jacobi method or the Gauss-Seidel method to a system of linear equations and obtain a divergent sequence of approximations. In such cases, it is said that the method diverges. So for convergence, the Diagonal Dominance of the matrix is necessary condition before applying any iterative methods. Moreover, also discussed about the error reduction factor in each iteration in Jacobi and Gauss-Seidel method.
\end{abstract}

Keywords: Jacobi Method, Gauss-Seidel Method, Convergence and Divergence, Diagonal Dominance, Reduction of Error.

\section{Introduction:}

An iterative technique to solve an $\mathrm{n} \times \mathrm{n}$ linear system $\mathrm{A} x=\mathrm{b}$ starts with an initial approximation $x_{0}$ to the solution $x *$, and generates a sequence of vectors $\left\{\mathrm{x}^{(\mathrm{k})}\right\}_{k=0}^{\infty}$ that converges to $x *$. Most of these iterative techniques involve a process that converts the system A $x=\mathrm{b}$ into an equivalent system of the form $x=\mathrm{S} x+\alpha$ for some $\mathrm{n} \times \mathrm{n}$ matrix $\mathrm{S}$ and vector $\alpha$. After the initial vector $x_{0}$ is selected, the sequence of approximate solution vectors is generated by computing $x^{(k+1)}=s^{(k)} x+\alpha, \mathrm{k}=1,2,3, \ldots \ldots$. The Jacobi method is based on solving for every variable locally with respect to the other variables; one iteration corresponds to solving for every variable once. It is easy to understand and implement, but convergence is slow.The Gauss-Seidel method is similar to the Jacobimethodexceptthatit uses updated valuesassoonastheyareavailable. ItgenerallyconvergesfasterthantheJacobi method, although still relatively slowly.

\subsection{Jacobi Method:}

The Jacobi method is a method of solving a matrix equation on a matrix that has no zeros along its main diagonal. Each diagonal element is solved for, and an approximate value put in. The process is then iterated until it converges.The Jacobi method is easily derived by examining each of the $\mathrm{n}$ equations in the linear system of equations $\mathrm{A} x=\mathrm{b}$. If in the $\mathrm{i}-$ th equation $\sum_{j=1}^{n} a_{i j} x_{j}=b_{i}$ solve for the value of $x_{i}$ while assuming the other entries of $x$ remain fixed. This gives

$$
x_{i}^{k+1}=\frac{1}{a_{i i}}\left[b_{i}-\sum_{j \neq i} a_{i j} x_{j}^{(k)}\right] \quad, \mathrm{i}=1,2, \ldots, \mathrm{n} .
$$

which is the Jacobi method. Note that thecomputation of $x_{i}^{(k+1)}$ requires each component in $x^{(k)}$ except $x_{i}^{(k)}$.In this method, the order in which the equations are examined is irrelevant, since the Jacobi method treats them independently.

The equation $\mathrm{A} x=\mathrm{b}$, which is $(\mathrm{L}+\mathrm{D}+\mathrm{U}) x=\mathrm{b}$, can be written asD $x=-(\mathrm{L}+\mathrm{U}) x+\mathrm{b}$. This reduces to $x_{i+1}=-$ $D^{-1}(L+U) x_{i}+D^{-1} b$

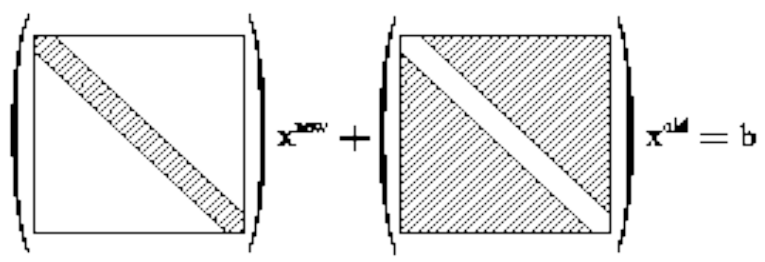

Fig. 1 : Splitting of matrix for Jacobi Method

Where $S_{j}=-D^{-1}(L+U)$ and $\alpha_{j}=D^{-1} b$

Note that if $\mathrm{A}$ is a strictly diagonally dominance matrix, then $\left\|S_{j}\right\|_{\infty}=\left\|-D^{-1}(L+U)\right\|_{\infty}<\left\|-D^{-1}\right\|_{\infty}\|(L+U)\|_{\infty}=\|D\|_{\infty}^{-1}\|(L+U)\|_{\infty}=\frac{\|(L+U)\|_{\infty}}{\max a_{i i}}<1$

Hence for any $x_{0} \in \mathrm{IRn}$, the sequence $\left\{\mathrm{x}^{(\mathrm{k})}\right\}_{k=0}^{\infty}$ defined by $x_{k}=S_{j} x_{k-1}+\alpha_{j} ;(\mathrm{k}=1,2, \cdots), \alpha_{j} \neq$ 0 converges to the unique solution of $x=S_{j} x+\alpha_{j}$. Therefore Jacobi's iterative technique will always guarantee a solution to the equation $\mathrm{A} x=\mathrm{b}$ whenever $\mathrm{A}$ is strictly diagonally dominant. 


\subsection{Gauss-Seidel Method:}

This method is modification of the Jacobi's iteration method. It is defined on matrices with non-zero diagonals, but convergence is only guaranteed if the matrix is either diagonally dominantor symmetric positive definite. The equation $\mathrm{A} x=\mathrm{b}$, which is $(\mathrm{L}+\mathrm{D}+\mathrm{U}) x=\mathrm{b}$, can be written as $(\mathrm{D}+\mathrm{L}) x=-\mathrm{U} x+\mathrm{b}$. This reducesto $x=-(D+L)^{-1} U x+(D+L)^{-1} b$

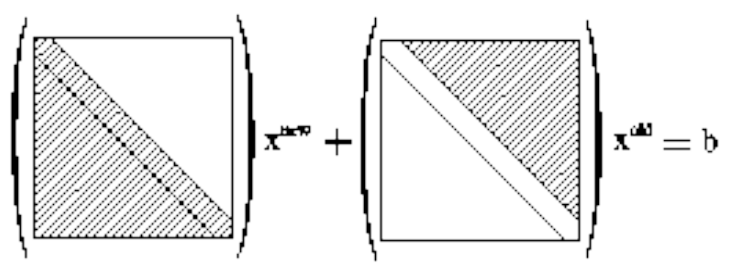

Fig. 2: Splitting of matrix for Gauss-Seidel

Method

Therefore $x^{(k+1)}=-(D+L)^{-1} U x^{(k)}+(D+L)^{-1}$

where $S_{g}=-(D+L)^{-1} U$ and $\alpha_{g}=(D+L)^{-1} b$

Now for solving the system of equations we use the iterative Gauss-Seidel method as given below $x_{i}^{(k+1)}=$ $\frac{1}{a_{i i}}\left[b_{i}-\sum_{j<i} a_{i j} x_{i}^{(k+1)}-\sum_{j \geq i} a_{i j} x_{i}^{(k)}\right], \quad \mathrm{i}=1,2,3, \ldots \ldots \ldots \ldots$

Note thatthecomputationof $x_{i}^{(k+1)}$ usesonlythoseelementsof $x^{(k+1)}$ thathavealreadybeencomputed $\left(x_{j}^{(k+1)}\right.$ for $j<$ $i$ ) and only those elements of $x^{(k)}$ that have yet to be advanced to iteration $\mathrm{k}+1,\left(x_{j}^{(k)}\right.$ for $\left.j \geq i\right)$. This means that no additional storage is required, and the computation can be donein place $\left(x^{(k+1)}\right.$ replaces $\left.x^{(k)}\right)$. While this might seem like a rather minor concern, for large systems it is unlikely that every iteration can be stored.If $\mathrm{A}$ is a strictly diagonally dominant matrix, then as we have done above in Jacobi iteration method, we get $\left\|S_{g}\right\|_{\infty}=\left\|-(D+L)^{-1} U\right\|_{\infty}<\left\|-(D+L)^{-1}\right\|_{\infty}\|(U)\|_{\infty}<1$.Thus for any $x_{0} \in \mathrm{IRn}$, the sequence $\left\{\mathrm{x}^{(\mathrm{k})}\right\}_{k=0}^{\infty}$ defined by $x_{k}=S_{g} x_{k-1}+\alpha_{g} ;(\mathrm{k}=1,2, \cdots) \quad, \alpha_{g} \neq 0$ converges to the unique solution of $x=S_{g} x+\alpha_{g}$, i.e., the Gauss-Seidel's iterative technique solves the equation $\mathrm{Ax}=\mathrm{b}$. Unlike in the Gauss-Seidel method, in Jacobi method, we can't overwrite $x_{i}^{(k+1)}$ with $x_{i}^{(k)}$ as that valuewillbeneededbytherestofthe computation. Thisisthemostmeaningful differencebetweenthe Jacobi and Gauss-Seidel methods.

\section{Importance of Diagonally Dominance:}

A square matrix $\mathbf{A}$ is diagonally dominant if $\left|\boldsymbol{a}_{i i}\right| \geq \sum_{j=1}^{n}\left|\boldsymbol{a}_{i j}\right|$ i.e., if the diagonal elements of each row exceeds in absolute value from sum of absolute values of all other entries in that row.If the inequality is strict then we say that the matrix is strictly diagonally dominant and if the inequality is greater than or equal to, then we can say that the matrix is weakly diagonal dominant .However, when these methods are combined with the more efficient methods, for example as a pre-conditioner, can be quite successful. For example see [3,4] ,It has been proved that if $\mathrm{A}$ is a strictly diagonally dominant (SDD) or irreducibly diagonally dominant, then the associated Jacobi and Gauss-Seidel iterations converge for any initial guess $x_{0}$ [4].If $\mathrm{A}$ is a symmetric positive definite (SPD) matrix, then the Gauss-Seidelmethod alsoconvergesfor any $x_{0}[1]$.If $\mathbf{A}$ is strictly diagonally dominant then $S_{j}=-D^{-1}(L+U)$ is convergent and Jacobi iteration will converge, otherwise the method will frequently converge.If $\mathbf{A}$ is not diagonally dominant then we must check $\rho\left(S_{j}\right)$ to see if the method is applicable and $\rho\left(S_{j}\right) \leq\left\|S_{j}\right\|$. If $\left\|S_{j}\right\|<1$ then $S_{j}$ is convergent and we use Jacobi's iteration method.But if $\rho\left(S_{j}\right)$ is nearer to unity then convergence will be slow.If the matrix is not diagonally dominant then the iterative methods Jacobi and Gauss-Seidel will diverge.

Example 2.1: Solve the linear system of equations

$$
2 x+y+6 z=9 ; 8 x+3 y+2 z=13 ; x+5 y+z=7
$$

Using initial approximation $(\mathrm{x}, \mathrm{y}, \mathrm{z})=(0,0,0)$ and show that both the methods diverge .

Solution: First, we rewrite the system of equations in the form

$\mathrm{x}=\frac{1}{2}(9-\mathrm{y}-6 \mathrm{z}) ; \mathrm{y}=\frac{1}{3}(13-8 \mathrm{x}-2 \mathrm{z}) ; \mathrm{z}=7-\mathrm{x}-5 \mathrm{y}$

now by using the Jacobi iteration formula (i) with initial approximation $(0,0,0)$ we get

$x^{(1)}=\frac{1}{2}(9-0-6 \times 0)=4.5$

$y^{(1)}=\frac{1}{3}(13-8 \times 0-2 \times 0)=4.333$

$z^{(1)}=7-0-5 \times 0=7$

as the first approximation. 
Convergence of Jacobi and Gauss-Seidel Method and Error Reduction Factor By repeated this we get the sequence of approximations as shown in the table :

\begin{tabular}{|c|c|c|c|c|c|c|}
\hline $\mathrm{n}$ & 0 & 1 & 2 & 3 & 4 & 5 \\
\hline $\mathrm{x}$ & 0 & 4.5 & -18.666 & 47.665 & -290.936 & 1220.284 \\
\hline $\mathrm{y}$ & 0 & 4.333 & -12.333 & 66.886 & -180.995 & 1030.226 \\
\hline $\mathrm{z}$ & 0 & 7 & -19.165 & 87.331 & -375.095 & 1202.911 \\
\hline
\end{tabular}

We can see in above table the Jacobi method become progressively worse instead of better. Thus we can conclude that the method diverges. Next we will see in Gauss-Seidel method, the given system of equations diverge faster than the Jacobi method .

By using the formula (ii) with initial approximation $(\mathrm{x}, \mathrm{y}, \mathrm{z})=(0,0,0)$ in (iii), we get

$x^{(1)}=\frac{1}{2}\left(9-y^{(0)}-6 z^{(0)}\right)=\frac{1}{2}(9-0-6 \times 0)=4.5$;

$y^{(1)}=\frac{1}{3}\left(13-8 x^{(1)}-2 z^{(0)}\right)=\frac{1}{3}(13-8 \times 4.5-2 \times 0)=-7.666$

$z^{(1)}=7-x^{(1)}-5 y^{(1)}=7-4.5-5 \times(-7.666)=40.833$

As first approximation.. By repeated this we get the sequence of approximations as shown in the table

\begin{tabular}{|c|l|l|l|l|l|}
\hline $\mathrm{n}$ & 0 & 1 & 2 & 3 & 4 \\
\hline $\mathrm{x}$ & 0 & 4.5 & -114.166 & 3723.535 & -120322.574 \\
\hline $\mathrm{y}$ & 0 & -7.666 & 281.554 & -9067.357 & 293117.698 \\
\hline $\mathrm{z}$ & 0 & 40.833 & -1286.604 & 41620.251 & -1345258.916 \\
\hline
\end{tabular}

Therefore neither the Jacobi method nor the Gauss-Seidel method converges to the solution of the system of linear equations.

2.2 Convergence of Jacobi and Gauss-Seidel method by Diagonal Dominance: Now interchanging the rows of the given system of equations in example 2.1, the system is

$$
8 x+3 y+2 z=13
$$

$$
\mathrm{x}+5 \mathrm{y}+\mathrm{z}=7
$$

$$
2 x+y+6 z=9
$$

is strictly diagonally dominant i.e. $|8|>|3|+|2| ;|5|>|1|+|1| ;|6|>|2|+|1|$

By applying Jacobi and Gauss-Seidel method we get the values given in the table below :

\section{Jacobi Iterations :}

\begin{tabular}{|l|l|l|l|l|l|l|l|l|}
\hline $\mathrm{n}$ & 0 & \multicolumn{1}{|c|}{1} & 2 & 3 & 4 & 5 & 6 & 7 \\
\hline $\mathrm{x}$ & 0 & 1.625 & 0.725 & 1.153 & 0.926 & 1.0385 & 0.9807 & 1.0098 \\
\hline $\mathrm{y}$ & 0 & 1.4 & 0.775 & 1.11 & 0.9436 & 1.0286 & 0.9855 & 1.0073 \\
\hline $\mathrm{z}$ & 0 & 1.5 & 0.725 & 1.129 & 0.9306 & 1.0340 & 0.9824 & 1.0088 \\
\hline
\end{tabular}

Gauss-Seidel Iterations :

\begin{tabular}{|l|l|l|l|l|l|l|}
\hline $\mathrm{n}$ & 0 & 1 & 2 & 3 & 4 & \multicolumn{1}{c|}{5} \\
\hline $\mathrm{x}$ & 0 & 1.625 & 1.027 & 0.9893 & 0.9973 & 0.9997 \\
\hline $\mathrm{y}$ & 0 & 1.075 & 1.0388 & 1.0052 & 1 & 0.9998 \\
\hline $\mathrm{z}$ & 0 & 0.779 & 0.9845 & 1.0027 & 1.0009 & 1.0001 \\
\hline
\end{tabular}

Thus we can conclude that the exact solution is $(\mathrm{x}, \mathrm{y}, \mathrm{z})=(1,1,1)$ because the error reduces in each proceeding iteration. Therefore strict diagonal dominance is a necessary condition for convergence of the Jacobi and Gauss-Seidel methods. No doubt Gauss Seidel method is much faster than the Jacobi method, it achieves more convergence in lesser number of iterations.

\section{Measurement of Reduction of Error:}

We consider the solution of linear system $\mathrm{Ax}=\mathrm{b}$ by the fixed point iteration Such iteration scheme can all be based on approximate inverse . By this mean any matrix $\mathrm{C}$ for which $\|I-C A\|<1$ in some matrix norm[2].Corresponding to an approximate inverse $\mathrm{C}$ for $\mathrm{A}$, we consider the iteration function $\mathrm{g}(\mathrm{x})=\mathrm{Cb}+(I-C A) x$

$$
=x+\mathrm{C}(b-A x)
$$

i.e., $\mathrm{g}(\mathrm{x})=\mathrm{x}-\mathrm{C} \mathrm{f}(\mathrm{x})$ where $\mathrm{f}(\mathrm{x})=\mathrm{Ax}-\mathrm{b}$

Also $\mathrm{g}(\mathrm{x})-\mathrm{g}(\mathrm{y})=\mathrm{Cb}+(I-C A) x-[\mathrm{Cb}+(I-C A) y]=(I-C A)(x-y)$

Consequently $\|\mathrm{g}(\mathrm{x})-\mathrm{g}(\mathrm{y})\| \leq\|(I-C A)\|\|x-y\| \quad$ with $\mathrm{K}=\|(I-C A)\|<1$ 
Therefore fixed point iteration $x^{(m+1)}=x^{(m)}+\mathrm{C}(b-A x) ; \mathrm{m}=0,1,2, \ldots \ldots \ldots$.

Starting from any $x^{(0)}$, will converge to unique solution $x$ with the error at each step reduces by at least a factor of $\mathrm{K}=\|(I-C A)\|$

3.1. Article:Suppose the matrix A is strictly row diagonally dominant i.e. $\left|\boldsymbol{a}_{i i}\right|>\sum_{j=1}^{n}\left|\boldsymbol{a}_{i j}\right|, j \neq i ; i=$ $1,2,3, \ldots \ldots, \mathrm{n}$.Let $\mathrm{D}=\operatorname{Diag}\left(a_{11}, a_{22}, a_{33}, \ldots \ldots \ldots, a_{n n}\right)$ be the diagonal of $\mathrm{A}$. Then $\left\|I-D^{-1} A\right\|_{\infty}={ }_{i}^{\max }\left\{1-\sum_{j}\left|a_{i j}\right| /\left|a_{i i}\right|\right\}$

$$
={ }_{i}^{\max }\left\{\sum_{j}\left|a_{i j}\right| /\left|a_{i i}\right|\right\}<1
$$

Showing that $\mathrm{D}$ is then an

approximate inverse of $\mathrm{A}$. The corresponding scheme $x^{(m+1)}=x^{(m)}+D^{-1}\left(b-A x^{(m)}\right) ; \quad \mathrm{m}=0,1,2,3, \ldots \ldots \ldots \ldots$ is Jacobi iteration.

In formulas Jacobi iteration discussed as $x_{i}^{(m)}=\frac{1}{a_{i i}}\left[b_{i}-\sum_{j \neq i} a_{i j} x_{j}^{(k)}\right], \mathrm{i}=1,2, \ldots, \mathrm{n}$.

Also Gauss-Seidel Iteration is defined as $x_{i}^{(k+1)}=\frac{1}{a_{i i}}\left[b_{i}-\sum_{j<i} a_{i j} x_{i}^{(k+1)}-\sum_{j \geq i} a_{i j} x_{i}^{(k)}\right], \forall i$

It is also known as the method of successive displacement .

For the linear system :

$10 \mathrm{x}+\mathrm{y}+\mathrm{z}=12 ; \mathrm{x}+10 \mathrm{y}+\mathrm{z}=12 ; \mathrm{x}+\mathrm{y}+10 \mathrm{z}=12$

Iterations starting with $x^{(0)}=0$, after applying both the methods, we get the values

\begin{tabular}{|c|c|c|c|c|c|c|}
\hline & \multicolumn{3}{|c|}{ Jacobi Method } & \multicolumn{3}{|c|}{ Gauss-Seidel Method } \\
\hline $\mathbf{m}$ & $\mathbf{x}$ & $\mathbf{y}$ & $\mathbf{z}$ & $\mathbf{x}$ & $\mathbf{y}$ & $\mathbf{z}$ \\
\hline 0 & 0 & 0 & 0 & 0 & 0 & 0 \\
\hline 1 & 1.2 & 1.2 & 1.2 & 1.2 & 1.08 & 0.972 \\
\hline 2 & 0.96 & 0.96 & 0.96 & 0.9948 & 1.0033 & 1.00019 \\
\hline 3 & 1.008 & 1.008 & 1.008 & 0.99965 & 1.000016 & 1.000033 \\
\hline 4 & 0.9984 & 0.9984 & 0.9984 & & & \\
\hline 5 & 1.00032 & 1.00032 & 1.00032 & & & \\
\hline 6 & 0.999936 & 0.999936 & 0.999936 & & & \\
\hline
\end{tabular}

We see that the sequences converges to the solution $(\mathrm{x}, \mathrm{y}, \mathrm{z})=(1,1,1)$ of the system.

Now $\left\|I-D^{-1} A\right\|_{\infty}={ }_{i}^{\max }\left\{\sum_{j \neq i}\left|a_{i j}\right| /\left|a_{i i}\right|\right\}={ }_{i}^{\max }\left\{\frac{1}{10}+\frac{1}{10}\right\}=0.2$

so that we would expect a solution in error by at least a factor of 0.2 per step, seen in above table.

\section{Conclusion:}

In this paper,we conclude that if the Jacobi iteration method converges for a given matrix A, then Gauss-Seidel method also converges and itgenerallyconvergesfasterthantheJacobi method but associated with condition that the matrix is strictly diagonal dominant.If $\|S\|<1$, then the iteration matrix $S$ is convergent and we use Jacobi's iteration method. But if $\rho(S)$ is nearer to unity then convergence is very slow.(that is, the spectral radius of iteration matrix $\mathrm{S}$ should be smaller). And at the end, we see that the error reduces in each step by the factor of $\mathrm{K}=\|(I-C A)\|$

\section{References:}

[1] Datta B N.Numerical Linear Algebra and Applications. Brooks/Cole Publishing Company,1995.

[2] Samuel D. Conte/Carl de Boor Elementary Numerical analysis An Algorithmic Approach, McGRAW-HILL INTERNATIONAL EDITION, Mathematics and statistics Series

[3] Li W. A note on the preconditioned Gauss Seidel (GS) method for linear system. J. Comput. Appl.Math., 2005, 182: 81-90.

[4] Saad Y. Iterative Methods for Sparse Linear Systems. PWS Press, New York, 1995 